\title{
Nonlinearity in Simple and Complex Cells in Early Biological Visual Systems
}

\begin{abstract}
The nonlinear model put forward in (Mahmoodi 2016) for early visual systems is investigated in details in this paper to explain some nonlinear behaviours of complex and some simple cells. Nonlinear cells are modelled as systems with linearnonlinear structures where the linear sub-unit is constructed by the layers proposed in (Mahmoodi 2016) and nonlinear sub-units are the results of an axon (modelled as a transmission line) carrying a series of spikes. In this paper, the nonlinear subsystems of complex cells are investigated in more details to show the mechanism by which nonlinear neurons work. Here the nonlinear systems modelling nonlinear sub-units of complex cells are represented by their first and second order responses. Our analytical as well as numerical results show good agreements with biological recordings reported in the literature.
\end{abstract}

\section{INTRODUCTION}

The scale space theory has been put forward as a linear model to describe early visual operations in a universal visual front end (Weickert et al. 1999; Lindeberg 2011). In this linear framework, a set of axioms for an early visual system are postulated to derive Gaussian kernels and their family as filters employed in the visual system (see e.g. (Weickert et al. 1997; Florack 1997; Ter Haar Romney 2003)). Florack et al. (Florack et al. 1992) have shown that the class of admissible scale-space kernels becomes limited by considering semigroup, scale invariance and rotational symmetry properties. In such a framework, a Gaussian kernel is derived for the visual system if the property of the spatial separability for the kernel in Cartesian coordinate systems is also added to the required characteristics for admissible scale space kernels. Gaussian scale space framework is also extended for temporal data 
(Koenderink 1988; Florack 1997; ter Haar Romney et al. 2001). Lindeberg at al. (Lindeberg and Fagerstrom 1996) then develop a time-recursive space time separable spatio-temporal scale space model. Duits et al. (Duits et al. 2004) also considers a Poisson scale space formulation in which the property of the local exterma non-enhancement is not included. Lindeberg (Lindeberg 2011; Lindeberg 2013) also presents a continuous time-scale model with a time recursive mechanism by exploiting a Gaussian scale space framework. In such a framework, a parametrization of the spatio-temporal filters with respect to image velocity and deformation is formulated. The fact that filters used in early biological visual systems are Gaussian related kernels, confirms the hypothesis that early biological visual systems possess the mathematical properties and axioms assumed for an early visual front end in scale-space community. A computational model based on RFs of LGN for orientation-selective cells in V1 of visual cortex is also presented in (Azzopardi and Petkov 2012). In this model, the RF of an orientation-selective neuron consists of a set of aligned RFs of LGN sub-units represented as the difference of Gaussians. The response of such an orientation-selective cell is then nonlinearly calculated as the weighted geometric mean of all sub-unit responses in the set. This model known as CORF exhibits the following three properties of simple cells: 1) contrast invariant orientation tuning, 2) cross orientation suppression and 3) response saturation. A push-pull inhibition is also included in this model in (Azzopardi et al. 2014) to demonstrate that the properties of simple cell that this push-pull model enjoys is extended to the relationship between spatial frequency and orientation tuning, and between spatial frequency tuning and contrast. Such relationships can be controlled by changing the strength of push-pull inhibition. On the other hand, a model for biological visual systems based on anatomical and physiological assumptions is proposed in (Mahmoodi 2016). This model is nonlinear in nature due to the way the signals are propagated through cascaded neurons. A linearity condition is found in (Mahmoodi 2016) to determine how such a visual system acts 
linearly. For situations where this system works linearly, its behaviour is in total agreement with scale space theory (Mahmoodi 2016). If the linearity condition, however, is not met, this visual system works nonlinearly. Initial attempts have been made in (Mahmoodi and Saba 2016) to explain the nonlinear behaviour of complex cells as well as some simple cells by using this model. However, the notion of higher order responses for nonlinear systems is not employed in (Mahmoodi and Saba 2016). The nonlinearity of the model presented in (Mahmoodi 2016) is also investigated here in this paper by incorporating the concepts of the first and second order responses of nonlinear systems to demonstrate that the nonlinear behaviour of the complex cells and some simple cells can indeed be explained by this visual model. Our contribution in this paper is therefore to explain how the nonlinear mechanism of the complex cells and nonlinear simple cells work by employing the model proposed in (Mahmoodi 2016) for early biological visual systems. Our paper is structured as follows: Section II describes the nonlinearity of the visual model investigated in this paper and demonstrates how this nonlinearity can account for the nonlinear cells' behaviour. Numerical results of the model investigated here are then compared with experimental recordings in biological literature in section III. Section IV finally draws the conclusions.

\section{NONLINEAR MODEL FOR NEURONS}

The model of the early visual system proposed in (Mahmoodi 2016) is nonlinear. This nonlinear system acts linearly under some certain conditions. The linear properties and linear receptive fields derived from this model are explored in (Mahmoodi 2016). In this paper, this nonlinear model is exploited to explain the behaviour of nonlinear complex cells in visual cortex. According to the model presented in (Mahmoodi 2016), the visual signal is propagated from retina to striate cortex through a series of cascaded neural layers. Each neural layer consists of interconnected neurons as shown in figure (1-top). Every neuron in 
each layer is also connected to a neuron in the next layer through its axon as shown in figure (1-bottom). Vertical and horizontal lines in figure (1-bottom) represent the axons and neural layers (consisting of dendrites) respectively. It is reported in the literature (see e.g. (Emerson et al. 1987; DeAngelis and Anzai 2004; DeAngelis et al. 1993)) that complex and some simple cells are nonlinear and have a linear- nonlinear $(\mathcal{L}-\aleph)$ structure. For these cells, the linear part is simply Gaussian-related receptive fields followed by non-linear static units possessing a nonlinear input/output relationship. For simple cells characterized with nonlinearity, the linear part consists of one linear sub-unit (kernel). This is different from complex cells which are characterized with parallel $(\mathcal{L}-\aleph)$ sub-units. According to the model presented in (Mahmoodi 2016), the neural layers are responsible for the linear subunits of the nonlinear cells. On the other hand, in this paper we demonstrate that the process of spikes propagating on axons behaving like transmission lines under some certain condition are responsible for the nonlinearity. In (Mahmoodi 2016), axons transmitting neural spikes from a neuron to another one are modelled as transmission lines. Neural spikes are also modelled as impulses (Dirac delta functions). As a result, the impulse response of an axon is calculated as (Mahmoodi 2016):

$$
v_{h}(z, t)=\frac{1}{2} \sqrt{\frac{1}{\pi t R_{z} C_{z}}} \exp \left(-\frac{G_{z} t}{C_{z}}\right) \exp \left(-\frac{R_{z} C_{z} z^{2}}{4 t}\right)
$$

where, $t, z, G_{z}, C_{z}$, and $R_{z}$ are the time that a spike reaches to the neuron in next layer from a neuron in the current layer, the length of axon, conductance, capacitance, and resistance of the axon (transmission line) per unit length respectively.

Obviously the response of the axon for a single spike propagated at $T_{n}$ and represented by $\delta\left(t-T_{n}\right)$ at $z$ can be written as: 


$$
v_{h}\left(z, t-T_{n}\right)=\frac{1}{2} \sqrt{\frac{1}{\pi\left(t-T_{n}\right) R_{z} C_{z}}} \exp \left(-\frac{G_{z}\left(t-T_{n}\right)}{C_{z}}\right) \exp \left(-\frac{R_{z} C_{z} z^{2}}{4\left(t-T_{n}\right)}\right)
$$

Membrane potentials on the dendrites greater than a threshold are transformed into a burst of spikes transmitted through the axon. For the cases where the delay of the latest spike (the spike with highest temporal distance from the beginning of the burst) in this burst is much less than the total time which spikes need to travel to the end of the axon (i.e. $\left.\max \left(T_{n}\right)<<t\right), v_{h}\left(z, t-T_{n}\right)$ in equation (2) is approximated as (Mahmoodi 2016):

$$
v_{h}\left(z, t-T_{n}\right) \approx \frac{1}{2} \sqrt{\frac{1}{\pi t R_{z} C_{z}}}\left(1+\frac{T_{n}}{2 t}\right) \exp \left(-\frac{G_{z}\left(t-T_{n}\right)}{C_{z}}\right) \exp \left(-\frac{R_{z} C_{z} z^{2}}{4 t}\left(1+\frac{T_{n}}{t}\right)\right)
$$

Or

$$
v_{h}\left(z, t-T_{n}\right)=v_{h}(z, t) \exp \left(a T_{n}\right)+\frac{T_{n}}{2 t} v_{h}(z, t) \exp \left(a T_{n}\right)
$$

where $a=\frac{G_{z}}{C_{z}}-\frac{R_{z} C_{z} z^{2}}{4 t^{2}}$. If a neuron (neuron A) sends $N$ spikes to another neuron in the next layer (neuron B), the potential accumulated in the neuron B's dendrites before neuron B starts firing is calculated as:

$$
v_{o}(z, t)=v_{h}(z, t) \sum_{n=1}^{N} \exp \left(a T_{n}\right)+\frac{v_{h}(z, t)}{2 t} \sum_{n=1}^{N} T_{n} \exp \left(a T_{n}\right)
$$

$T_{n}$ can be written as a sum of all time intervals between consecutive spikes, i.e.:

$$
T_{n}=\sum_{p=1}^{n} \Delta T_{p}=n \bar{T}
$$

where $\bar{T}$ is the mean time interval between consecutive spikes. Equation (5) can therefore be rewritten as: 


$$
v_{o}(z, t)=v_{h}(z, t) \exp (a \bar{T})\left(\frac{\exp (a \bar{T} N)-1}{\exp (a \bar{T})-1}-\frac{\bar{T}}{2 t} \frac{N \exp (a \bar{T} N)-(N+1) \exp (a \bar{T} N)}{(\exp (a \bar{T})-1)^{2}}\right)
$$

The mean time interval between consecutive spikes, $\bar{T}$, is assumed to be much smaller than the time needed for spikes to reach to the end of the axon, $t$, therefore $v_{o}(z, t)$ can be approximated as:

$$
v_{o}(z, t) \cong v_{h}(z, t) \exp (a \bar{T})\left(\frac{\exp (a \bar{T} N)-1}{\exp (a \bar{T})-1}\right)
$$

where $N$ is the total number of spikes in the burst. According to classical rectification model (Carandini and Fester 2000) for neural firing rate, the input membrane potential of neuron A is proportional to firing rate and therefore $N$. The classical rectification model (Carandini and Fester 2000) indicates that the firing rate and therefore $N$ increases linearly with respect to the membrane potential $v_{i n}$, i.e.: $N=k v_{i n}$, where $k$ is a constant. In other words, equation (8) is written as:

$$
v_{o}(z, t) \cong v_{h}(z, t) \exp (a \bar{T})\left(\frac{\exp \left(k a \bar{T} v_{i n}\right)-1}{\exp (a \bar{T})-1}\right)
$$

Equation (9) shows the input-output relationship for a neuron behaving nonlinearly. As seen from this equation, this nonlinearity is in a form of an exponential function. It is interesting to note that the input-output relationship of some neurons represented by equation (9) is similar to the input-output relationship of an electrical diode. In this section, we therefore demonstrate how such neurons can be exploited in the early visual circuitry to implement complex cells and some nonlinear simple cells. But before this, let us explore the possibility of a neuron whose input-output relationship is governed by equation (9), to work like a linear amplifier. 


\section{II.A) NEURAL Signal AMPLIFICATION}

In order to see the amplification ability of neurons possessing the input-output relations similar to (9), let us assume that the input voltage consists of two components: one which is constant $V_{\text {in }}$ over a certain period of time and the other one which has small values $v_{i}$ with respect to $V_{\text {in }}$, i.e.: $v_{\text {in }}=V_{\text {in }}+v_{i}$. The output $v_{o}$ can then be approximated as:

$$
v_{o}(z, t) \cong v_{h}(z, t) \frac{\exp (a \bar{T})}{\exp (a \bar{T})-1}\left(\exp \left(k a \bar{T}\left(V_{i n}+v_{i}\right)\right)-1\right) \approx \frac{v_{h}(z, t) \exp (a \bar{T})}{\exp (a \bar{T})-1}\left(\left(1+k a \bar{T} v_{i}\right) \exp \left(k a \bar{T} V_{i n}\right)-1\right)
$$

As observed from equation (10), the relationship between $v_{i}$ and $v_{o}$ is linear for small values of $v_{i}$. In a linear circuit, the ratio of the output voltage to input voltage, $\frac{v_{o}}{v_{i}}$, is known as the electrical gain of the linear circuit. For $v_{i}<<V_{i n}$, the neuron represented with equation (9) acts linearly with respect to $v_{i}$ as described by equation (10). Therefore, an electrical gain can be defined for this neuron. Such an electrical gain is proportional to $\frac{v_{h}(z, t) k a \bar{T} \exp (a \bar{T})}{\exp (a \bar{T})-1}$. The condition under which this linear circuit amplifies the input signal is:

$$
\begin{aligned}
& \frac{v_{h}(z, t) k a \bar{T} \exp (a \bar{T})}{\exp (a \bar{T})-1}>1 \\
& \text { or } \\
& v_{h}(z, t) k a \bar{T}>1
\end{aligned}
$$

In the above inequalities, $z$ and $t$ are as usual, the axon length, and the time it takes a spike to travel to the end of the axon. In practice, to satisfy the pre-condition $v_{i}<<V_{\text {in }}$, there 
should be enough background illumination (producing $V_{i n}$ ) in the visual scene in steady state so that changes in illumination ( responsible for $v_{i}$ ) inside the visual scene is small in comparison with the background illumination. This is inline with the fact that our visual system fails to recognise the colours in a dark room. Here this dark room experience could be interpreted as the case where there is no linear electrical amplification of input signals (voltages) due to the small values for $V_{i n}$.

\section{II.B) UNITS WITH INPUT-OUTPUT RELATIONSHIP $v_{o}=v_{i n}^{n}$}

Biological recordings show that the input-output relationships of most nonlinear cells such as some simple neurons have a static unit with a form of $v_{o}=v_{i n}^{n}$ (DeAngelis and Anzai 2004; DeAngelis et al. 1993).

Units whose input-output relationships are represented by equation (9) demonstrate an input-output characteristic similar to an electrical diode. Figure (2) shows a circuit diagram based on $\log$ and anti-log amplifiers in which sub-units with label $D$ represent a system with the input-output relationship determined by equation (9). These sub-units are used to implement a circuit with input-output relationship of the form $v_{o}=v_{i n}^{n}$ as shown in figure (2). In fact, these sub-units $D$ represent the axons of some neurons acting nonlinearly according to equation (9). The arrow shape in one end of the sub-unit $D$ in figure (2) represents the output of the sub-unit. Therefore, the other end (the flat end in figure (2)) of the sub-unit corresponds to the input of the sub-unit. The left sub-unit with amplifier $\mathrm{A}$ in figure(2) constitutes a logarithm function which simply obtains the logarithm of the input signal. Amplifier B in this figure is a linear amplifier (such as the one described in section II.A) with a voltage gain of $n$. Finally, the right sub-unit D parallel with Amplifier C in this figure is an exponential function, which obtains the exponential value of its input. The total 
output-input relationship then becomes of the form of $v_{o}=v_{i n}^{n}$. Biological data from nonlinear neurons possessing static nonlinearity of the form $v_{o}=v_{i n}^{n}$ demonstrates that the value of $n$ may vary between unity and 3.2 in a population of nonlinear neurons (DeAngelis and Anzai 2004). However a majority of these nonlinear neurons are characterized with a value of 2 for $n$ (DeAngelis and Anzai 2004). Therefore the input-output relationship of the static unit in a majority of these nonlinear neurons is of the form $v_{o}=v_{i n}^{2}$. The model presented in figure (2) is also in line with the biological recordings reported in (DeAngelis and Anzai 2004; DeAngelis et al. 1993) (see e.g figure 44.5 and the related text in (DeAngelis and Anzai 2004)) to measure directional similarity index. In the experiment presented in (DeAngelis et al. 1993; DeAngelis and Anzai 2004), the exponents of the nonlinear cells vary in range between 0.5 to 8 . The gain of linear amplifier B shown in figure (2) can vary in that range and it is this gain which determines the exponent of the nonlinear unit with $v_{o}=v_{i n}^{n}$ input-output relationship.

\section{II.C) NONLINEAR NEURONS}

The linear-nonlinear $(\mathcal{L}-\aleph)$ structure of complex and some simple cells in biological literature (see e.g. (Emerson et al. 1987; DeAngelis and Anzai 2004; DeAngelis et al. 1993)) consists of a linear receptive field followed by a nonlinear sub-unit whose input-output relationship is of the form of $v_{o}=v_{i n}^{n}$. The output of the linear receptive field is the input to the nonlinear sub-unit. Nonlinear simple cells are characterized with a $\mathcal{L}-\aleph$ structure in which a single linear receptive field is followed by a nonlinear unit (DeAngelis and Anzai 2004). Complex cells, on the other hand, consist of parallel $\mathcal{L}-\aleph$ sub-units whose outputs are summed together to form the final output (Adelson and Bergen 1985; DeAngelis and Anzai 2004). 


\section{II.D) $\mathcal{L}-\mathrm{S}$ SYSTEM IDENTIFICATION}

Linear-nonlinear-linear $(\mathcal{L}-\aleph-\mathcal{L})$ systems are widely used in biology to identify the systems of nonlinear neurons (see e.g. (Spekreijse 1969; Korenberg 1973; Korenberg and Hunter 1986)). Nonlinear neurons similar to any nonlinear system are represented and identified with higher order kernels such as Wiener or Volterra kernels (Wiener 1958; Volterra 1959). For a system consisting of cascaded linear-nonlinear-linear sub-units each Wiener kernel is directly proportional to the Volterra kernel of the corresponding order (Korenberg and Hunter 1986). If the nonlinearity in this cascade is described by a polynomial, i.e.:

$$
v=\sum_{i=0}^{I} a_{i} u^{i}
$$

Where $v=v_{o}$ and $u=v_{i n}$. Then the first order Volterra kernel is calculated as (Korenberg and Hunter 1986):

$$
h_{1}(t)=a_{1} \int_{0}^{+\infty} k(\tau) g(t-\tau) d \tau
$$

where $a_{1}$ is a non-zero constant coefficient in equation (11) and $k(\tau)$ and $g(\tau)$ are the impulse responses of the linear subsystems inside the cascade before and after the nonlinear subsystem as shown in figure (3).

Similarly the second order Volterra kernel is calculated as (Korenberg and Hunter 1986):

$$
h_{2}\left(t_{1}, t_{2}\right)=a_{2} \int_{0}^{+\infty} k(\tau) g\left(t_{1}-\tau\right) g\left(t_{2}-\tau\right) d \tau
$$


where again $a_{2}$ is a non-zero constant coefficient in equation (11). It is reported in biological literature that only second order kernels are sufficient to represent nonlinear neurons in the early visual system (DeAngelis and Anzai 2004). Here, we therefore employ up to only second order responses for nonlinear cells in this paper. Here, we exploit the aforementioned $\mathcal{L}-\aleph-\mathcal{L}$ system identification framework for $\mathcal{L}-\aleph$ system identification associated with the nonlinear neurons discussed earlier in this paper. To this end, let us consider $k(\tau)=\delta(\tau)$ where $\delta(\tau)$ is a Dirac delta function. In such a case, one can write:

$$
y(t)=\delta(t) * v(t)=v(t)
$$

where $y$ and $v$ are the output signals of the cascaded system and the nonlinear subsystem respectively as shown in figure (3). In this case, the $\mathcal{L}-\boldsymbol{\aleph}-\mathcal{L}$ system is reduced to a $\mathcal{L}-\boldsymbol{\aleph}$ system. Consequently, equations (12) and (13) are changed to equations (15) and (16):

$$
\begin{aligned}
& h_{1}(t)=a_{1} g(t) \\
& h_{2}\left(t_{1}, t_{2}\right)=a_{2} g\left(t_{1}\right) g\left(t_{2}\right)
\end{aligned}
$$

Neurons are characterized with spatial as well as temporal profiles. Equations (12) and (13) for $\mathcal{L}-\aleph-\mathcal{L}$ systems should therefore be extended and written with respect to both spatial and temporal domains, i.e.:

$$
\begin{gathered}
h_{1}(x, y, t)=a_{1} \iiint k\left(x^{\prime}, y^{\prime}, \tau\right) g\left(x-x^{\prime}, y-y^{\prime}, t-\tau\right) d x^{\prime} d y^{\prime} d \tau \\
h_{2}\left(x_{1}, x_{2}, y_{1}, y_{2}, t_{1}, t_{2}\right)=a_{2} \iiint k\left(x^{\prime}, y^{\prime}, \tau\right) g\left(x_{1}-x^{\prime}, y_{1}-y^{\prime}, t_{1}-\tau\right) g\left(x_{2}-x^{\prime}, y_{2}-y^{\prime}, t_{2}-\tau\right) d x^{\prime} d y^{\prime} d \tau
\end{gathered}
$$

Similar to the treatments for equations (15) and (16), the first and second order kernels for $\mathcal{L}-\aleph$ systems can be calculated by assuming $k(x, y, \tau)=\delta(x, y, \tau)$, i.e.: 


$$
\begin{aligned}
& h_{1}(x, y, t)=a_{1} g(x, y, t) \\
& h_{2}\left(x_{1}, x_{2}, y_{1}, y_{2}, t_{1}, t_{2}\right)=a_{2} g\left(x_{1}, y_{1}, t_{1}\right) g\left(x_{2}, y_{2}, t_{2}\right)
\end{aligned}
$$

It is usually more convenient to write equation (20) in terms of $\Delta x=x_{2}-x_{1}$, $\Delta y=y_{2}-y_{1}$, and $\Delta t=t_{2}-t_{1}$, i.e.:

$$
h_{2}\left(x_{1}, x_{1}+\Delta x, y_{1}, y_{1}+\Delta y, t_{1}, t_{1}+\Delta t\right)=a_{2} g\left(x_{1}, y_{1}, t_{1}\right) g\left(x_{1}+\Delta x, y_{1}+\Delta y, t_{1}+\Delta t\right)
$$

Complex cells are considered to possess a structure consisting of parallel systems (Adelson and Bergen 1985) as shown in figure (5). If the input-output relationships for $m($.$) and p($.$) nonlinear subsystems shown in figure (5) are written in a form of$ polynomials as:

$$
\begin{aligned}
& v_{o m}=\sum_{i=0}^{I} a_{i} v_{i n m}^{i} \\
& v_{o p}=\sum_{i=0}^{I} b_{i} v_{i n p}^{i}
\end{aligned}
$$

Then the first and second order Volterra kernels are written as:

$$
\begin{aligned}
& h_{1}(x, y, t)=a_{1} g(x, y, t)+b_{1} f(x, y, t) \\
& h_{2}\left(x_{1}, x_{2}, y_{1}, y_{2}, t_{1}, t_{2}\right)=a_{2} g\left(x_{1}, y_{1}, t_{1}\right) g\left(x_{2}, y_{2}, t_{2}\right)+b_{2} f\left(x_{1}, y_{1}, t_{1}\right) f\left(x_{2}, y_{2}, t_{2}\right)
\end{aligned}
$$

Or 


$$
\begin{aligned}
& h_{2}\left(x_{1}, x_{1}+\Delta x, y_{1}, y_{1}+\Delta y, t_{1}, t_{1}+\Delta t\right)=a_{2} g\left(x_{1}, y_{1}, t_{1}\right) g\left(x_{1}+\Delta x, y_{1}+\Delta y, t_{1}+\Delta t\right) \\
& +b_{2} f\left(x_{1}, y_{1}, t_{1}\right) f\left(x_{1}+\Delta x, y_{1}+\Delta y, t_{1}+\Delta t\right)
\end{aligned}
$$

where $f(x, y, t)$ and $g(x, y, t)$ are the impulse responses of linear subsystems in parallel. In the next section, we exploit equations (24) to (26) with conditions (22) and (23) to calculate the second order Volterra (and therefore Wiener) kernels for biological visual systems modelled as $\mathcal{L}-\boldsymbol{N}$ systems and therefore compare our simulation results with the biological recordings.

\section{RESULTS}

In this section, the simulation results obtained from our model described in section II are presented and compared with the biological recordings reported in the literature. It is reported in biology that nonlinear simple cells are characterized with a half-squaring property as a nonlinear system. As indicated in section II.B, figure (2) shows a circuit diagram producing a powering relationship (function) between input and output of the subsystem. The subsystem presented in figure (2) consists of an amplifier (amplifier B) with the gain of $n$ sandwiched between a log and anti-log amplifier. It is easy to see that the input membrane potential of a neuron appears as the firing frequency of spikes in equation (8) and consequently equation (9). The input-output relationship of the circuit diagram presented in figure (2) is of the form $v_{o}=v_{i n}^{n}$. It is reported in the biological literature that $n$ is around two (DeAngelis and Anzai 2004). Since frequency is never negative, the input-output relationship of a subsystem described in figure (2) for $n=2$ is a half-squaring function which is in total agreement with biological recordings presented in (DeAngelis and Anzai 2004) and shown in figure (4). 
According to energy model (Adelson and Bergen 1985), complex cells contain parallel linear-nonlinear subsystems as depicted in figure (5). In this paper, we argue that the nonlinear sub-unit of nonlinear neurons such as complex cells exists due to the process of the propagation of a series of spikes propagating through the cell's axon behaving like a transmission line (see section II). This is to say that a complex cell could consist of some simple cells whose nonlinear sub-unit is their axon behaving nonlinearly in the presence of neural spikes. Here we expand equation (9) by using Taylor's series for the nonlinear subunit $m($.$) shown on the top of figure (5) to obtain the nonlinear input-output relationship in a$ form of polynomial expansions such as equations (22) and (23).

$$
v_{m o}(z, t) \cong v_{m h}(z, t) \frac{\exp \left(a_{m} \bar{T}_{m}\right)}{\exp \left(a_{m} \bar{T}_{m}\right)-1}\left(b_{m 1} v_{i n m}+\frac{1}{2} b_{m 2} v_{i n m}^{2}+\frac{1}{6} b_{m 3} v_{i n m}^{3}+\ldots\right)
$$

where $b_{m i}=\left(a_{m} k_{m} \bar{T}_{m}\right)^{i}$ and, $a_{m}$ and $T_{m}$ are constants discussed in the previous section and related to the nonlinear sub-unit $m($.$) in the complex cell. Similarly for the nonlinear sub-$ unit $p($.$) , we can write:$

$$
v_{p o}(z, t) \cong v_{p h}(z, t) \frac{\exp \left(a_{p} \bar{T}_{p}\right)}{\exp \left(a_{p} \bar{T}_{p}\right)-1}\left(b_{p 1} v_{i n p}+\frac{1}{2} b_{p 2} v_{i n p}^{2}+\frac{1}{6} b_{p 3} v_{i n p}^{3}+\ldots\right)
$$

where $b_{p i}=\left(a_{p} k_{p} \bar{T}_{p}\right)^{i}$ and, $a_{p}$ and $T_{p}$ are constants associated with the nonlinear subsystem $p($.$) in the complex cell. Equations (27) and (28) are in the form of the conditions$ (22) and (23). According to equations (24) to (26), the first and second order kernels can therefore be written as:

$$
\begin{aligned}
& h_{1}(x, y, t)=B_{m 1} g(x, y, t)+B_{p 1} f(x, y, \tau) \\
& h_{2}\left(x_{1}, x_{2}, y_{1}, y_{2}, t_{1}, t_{2}\right)=B_{m 2} g\left(x_{1}, y_{1}, t_{1}\right) g\left(x_{2}, y_{2}, t_{2}\right)+B_{p 2} f\left(x_{1}, y_{1}, t_{1}\right) f\left(x_{2}, y_{2}, t_{2}\right)
\end{aligned}
$$


where

$$
\begin{aligned}
& B_{m 1}=v_{m h}(z, t) \frac{\exp \left(a_{m} \bar{T}_{m}\right) b_{m 1}}{\exp \left(a_{m} \bar{T}_{m}\right)-1} \\
& B_{m 2}=\frac{v_{m h}(z, t) \exp \left(a_{m} \bar{T}_{m}\right) b_{m 2}}{2\left(\exp \left(a_{m} \bar{T}_{m}\right)-1\right)} \\
& B_{p 1}=v_{p h}(z, t) \frac{\exp \left(a_{p} \bar{T}_{p}\right) b_{p 1}}{\exp \left(a_{p} \bar{T}_{p}\right)-1} \\
& B_{p 2}=\frac{v_{p h}(z, t) \exp \left(a_{p} \bar{T}_{p}\right) b_{p 2}}{2\left(\exp \left(a_{p} \bar{T}_{p}\right)-1\right)}
\end{aligned}
$$

As an example, for a complex cell, the impulse responses $g$ and $f$ in equations (29) and (30) and figure (5) can be:

$$
\begin{aligned}
& g(x, y, z, t) \sim K_{n}(x, y) v_{h}(t ; z) \\
& f(x, y, z, t) \sim \frac{\partial^{3} K_{n}(x, y)}{\partial x^{3}} \frac{\partial v_{h}(t ; z)}{\partial t}
\end{aligned}
$$

where $v_{h}(t ; z)$ is given in equation (1) and $K_{n}(x, y)=K_{n-1}(x, y) * K(x, y)$ in which $K(x, y)$ is the modified Bessel function of the second kind and zero degree (see section 2.3 in (Mahmoodi 2016) for more details about $K_{n}(x, y)$ and $\left.v_{h}(t ; z)\right)$. In the early visual system, the outputs of a few simple cells can be the inputs to the complex cell. These simple cells can be, for example, spatially arranged in the way shown in

1) red circles of figure (1-top) for complex cells with elongated non-isotropic second order response (see section 3.3 in (Mahmoodi 2016) for elongated cells for more details) or 
2) blue and red circles in figure (1-bottom) for complex cells with directional selectivity in their second order response (see section 3.4 in (Mahmoodi 2016) for cells with directional selectivity for more details ).

For complex cells with elongated second order response, our numerical result shown in figure (6-a) and computed by using equation (21) is in good agreement with the biological recording shown in figure (6-b) and reported in (DeAngelis and Anzai 2004). In the case of a nonlinear complex cell with directional selectivity appearing in its second order response, the first and second order spatio-temporal responses are shown in figure (7-a and b). For such a complex cell, $B_{m 1}>>B_{p 1}$ and $B_{m 2}<<B_{p 2}$. Figures (7-c and d) also show the first and second order spatio-temporal responses recorded for a complex cell in biological experiments (DeAngelis and Anzai 2004). As can be seen from this figure, the second order spatiotemporal response of the complex cell predicts a directional selectivity; however, the first order response shows no directional selectivity. Our simulation results shown in this figure is therefore in total agreement with experimental results recorded in biological literature. Let us now investigate the effects of coefficients such as $B_{m 1}, B_{m 2}, B_{p 1}$ and $B_{p 2}$ in the first and second order responses of a complex cell. To this end, we assume that the spatial impulse responses of the linear subsystems of a complex cell with parallel sub-units and an $\mathcal{L}$ structure similar to the one depicted in figure (5), are shown in figures (8-a) and (8-b) respectively. The three neurons in the tenth layer are arranged as the red circles shown in figure (1-top). The outputs of these three neurons are the inputs of a complex cell whose first and second spatial responses are determined by equations (29) and (30). Figure (9) shows the first order spatial response determined by equation (29) for six different values of $\frac{B_{p 1}}{B_{m 1}}$ (see the caption of figure (9) for more details). As can been seen from figures (9), the first order spatial response corresponds to the spatial impulse response of one of linear subsystems for 
$0 \leq \frac{B_{p 1}}{B_{m 1}} \leq \frac{1}{10}$ (figures (9-a) and (9-b)). Figures (9-c) to (9-f) also show other scenarios where the first order spatial response is a combination of the two spatial impulse responses of the linear sub-units. The second order spatial responses given by equation (30), are also depicted in figure (10) for six different values of the ratio $\frac{B_{m 2}}{B_{p 2}}$. As observed from this figure, for $0 \leq \frac{B_{m 2}}{B_{p 2}} \leq \frac{1}{10}$, the second order spatial responses shown in figures $(10-\mathrm{a})$ and $(10-\mathrm{b})$ are very similar to one of the spatial impulse responses of the linear subsystems of the complex cell shown in figure (8). Other second order spatial responses determined in equation (3) and depicted in figures (10-c) to (10-f) are a linear combination of the two spatial impulse responses. Figures (11) and (12) illustrate the similar results for the first and second order spatiotemporal responses of a complex cell with an $\mathcal{L}-\aleph$ structure in which the spatiotemporal impulse responses of the linear subsystems are depicted in figures (7-a) and (7-b). As can be observed from figures (11) and (12) and similar to the cases in figures (9) and (10), the first and the second order spatiotemporal responses of a complex cell are similar to one of the spatiotemporal impulse responses of the linear subsystems when $0 \leq \frac{B_{p 1}}{B_{m 1}} \leq \frac{1}{10}$ and $0 \leq \frac{B_{m 2}}{B_{p 2}} \leq \frac{1}{10}$ in equations (29) an (30). The recorded data from biological experiments shown in figures (7-a) and (7-b) suggest that the range of ratios $\frac{B_{p 1}}{B_{m 1}}$ and $\frac{B_{m 2}}{B_{p 2}}$ is expected to be in the interval of $\left[0 \frac{1}{10}\right]$. 


\section{CONCLUSIONS}

The nonlinearity of a visual model proposed in the previous work (Mahmoodi 2016) is investigated in this paper. This nonlinearity exists due to axons behaving like a transmission line at the presence of neural spikes. It is demonstrated here that the characteristics of nonlinear simple cells and also complex cells can be explained by this nonlinearity. By using the $\mathcal{L}-\kappa$ configuration, the first order and second order responses of the nonlinear simple cells and complex cells are explained by the model considered in this paper. The nonlinear unit of this $\mathcal{L}-\boldsymbol{N}$ system is associated with the transmission line behaviour of the axons of the cells. Our formulations here show that such a nonlinear sub-unit behaves in a similar fashion to an electrical diode. It is discussed here that such a nonlinear element can be configured to work linearly and therefore can be used as a linear amplifier in neural circuits. Our formulations here also demonstrate why complex cells may not show any directional selectivity in their first order response but their second order response demonstrates a directional selectivity.

\section{REFERENCES}

Adelson EH, Bergen JR, (1985) Spatiotemporal Energy Models for the Perception of Motion, Journal of Optical Society of American Association, Vol. 2, pp:284-299,.

Azzopardi G, Petkov N, (2012) A CORF Computational Model of a Simple Cell that Relies on LGN Input Outperforms the Gabor Function Model, Biological Cybernetic, Vol. 106, pp:177-189.

Azzopardi G, Rodriquez-Sanchez A, Piater J., Petkov, N, (2014), A Push-Pull CORF Model of a Simple Cell with Antiphase Inhibition Improves SNR and Contour Detection, PLOS ONE, Vol. 9, No. 7, pp 1-13. 
Carandini M, Fester D, (2000) Membrane Potential and Firing Rate in Cat Primary Visual Cortex, Journal of Neuroscience, Vol. 20, No. 1, pp:470-484.

DeAngelis GC, Anzai A, (2004) A Modern View of the Classical Receptive Field: Linear and Non-Linear Spatiotemporal Processing by V1 Neurons, In L.M. Chalupa and J.S. Werner (eds.), The Visual Neuroscience, Vol. 1, MIT Press, Cambridge, pp. 704-719.

DeAngelis GC, Ohzawa I, Freeman RD, (1993) Spatiotemporal Organization of Simple Cell Receptive Fields in the Cat's Striate Cortex. II. Linearity of Temporal and Spatial Summation, Journal of Neurophysiology, Vol. 69, No. 4, pp: 1118-1135.

Duits R, Florack L, de Graaf J, ter Haar Romeny B, (2004) On the Axioms of Scale Space Theory, Journal of Mathematical Imaging and Vision, Vol. 22, pp. 267-298.

Emerson RC, Citron MC, Vaughn WJ, Klein SA, (1987) Nonlinear Directionally Selective Sub-units in Complex Cells of Cat Striate Cortex, Journal of Neurophysiology, Vol. 58, No. 1, pp:33-65.

Florack L, ter Haar Romney B, JKoenderink J, Viergever M, (1992) Scale and the Differential Structure of Images, Image and Vision Computing, Vol. 10, No. 6, pp. 376388.

Florack L., (1997) Image Structure, Series in Mathematical Imaging and Vision, Springer, Dordrecht.

Koenderink JJ, (1998) Scale-Time, Biological Cybernetic, Vol. 58, pp. 159-162.

Korenberg MJ, (1973) Identification of Biological Cascades of Linear and Static Nonlinear Systems, Proceedings of Midwest Symposium Circuit Theory, Vol. 18, No. 2, pp:1-9.

Korenberg MJ, Hunter LW, (1986) The Identification of Nonlinear Biological Systems: LNL Cascade Models, Biological Cybernetic, Vol. 55, pp:125-134. 
Lindeberg T, Fagerstrom D, (1996) Scale-Space with Causal Time Direction, In: Proceedings of $4^{\text {th }}$ European Conference on Computer Vision, Vol. 1064, pp. 229-240, Springer, Berlin.

Lindeberg T, (2011) Generalized Gaussian Scale-Space Axiomatic Comprising Linear ScaleSpace, Affine Scale-Space and Spatio-Temporal Scale-Space Journal of Mathematical Imaging and Vision, Vol. 40, pp: 36-81.

Lindeberg T, (2013) A Computational Theory of Visual Receptive Fields, Biological Cybernetics, Vol. 107, pp: 589-635.

Mahmoodi S, (2016) Linear Neural Circuitry Model for Visual Receptive Fields, Journal of Mathematical Imaging and Vision, Vol. 54, No. 2, pp. 138-161,.

Mahmoodi S, Saba N (2016) Nonlinear Model for Complex Neurons in Biological Visual Systems, $9^{\text {th }}$ International Conference on Bio-Inspired Systems and Signal Processing, Rome, 6 pages.

Spekreijse H, (1969) Rectification in the Goldfish Retina: Analysis by Sinsoidal and Auxiliary Stimulation Vision Research, Vol. 9, pp: 1461-1472.

ter Haar Romeny B, Florack L, Nielsen M, (2001) Scale-time Kernels and Models, In: ScaleSpace and Morphology, Proceedings of Scale-Space'01, Vancouver, Canada, LNCS, Springer, Berlin.

ter Haar Romeny B, (2003) Front-End Vision and Multi-Scale Image Analysis”. Springer Dordrecht.

Volterra V, (1959) Theory of Functionals and of Integral and Integro-Differential Equations, New York, Dover.

Weickert J, Ishikawa S, Imiya A, (1997) On the History Gaussian Scale-Space Axiomatic, chapter 4, pp.45-59, in J. Sporring, M. Nielsen, L.M.J. Florack and P. Johansen (Eds.), Gaussian Scale Space Theory, Vol. 8, Computational Imaging and Vision Series, Kulwer. 
Weickert J, Ishikawa S, Imiya A, (1999) Linear Scale Space has First been proposed in Japan, Journal of Mathematical Imaging and Vision, Vol. 10, pp. 237-252.

Wiener N, (1958) Nonlinear Problems in Random Theory, Massachusetts, MIT Press. 

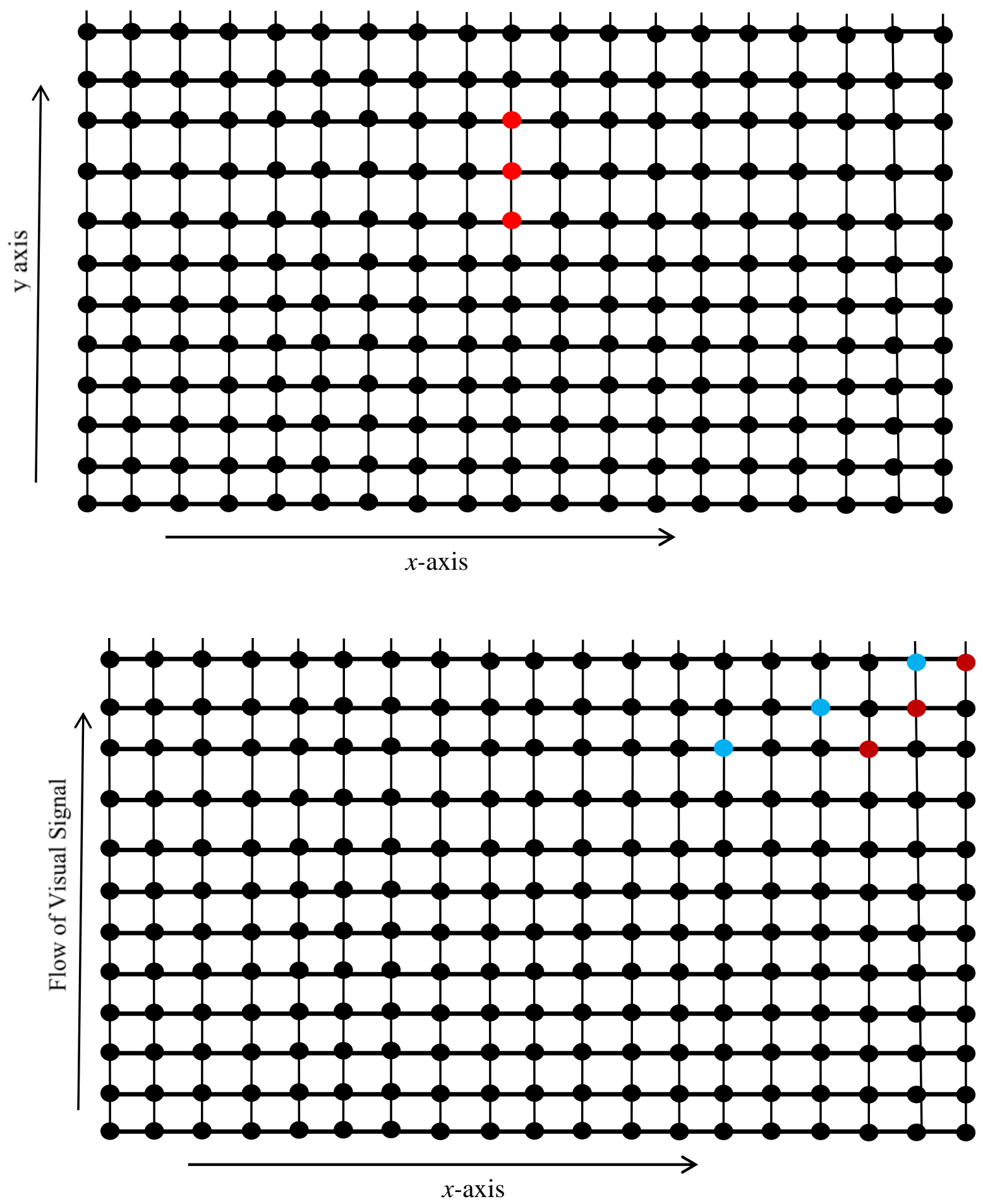

Figure (1): (top) A layer consists of interconnected neurons (bottom) A cross-section of a series of neural layers 


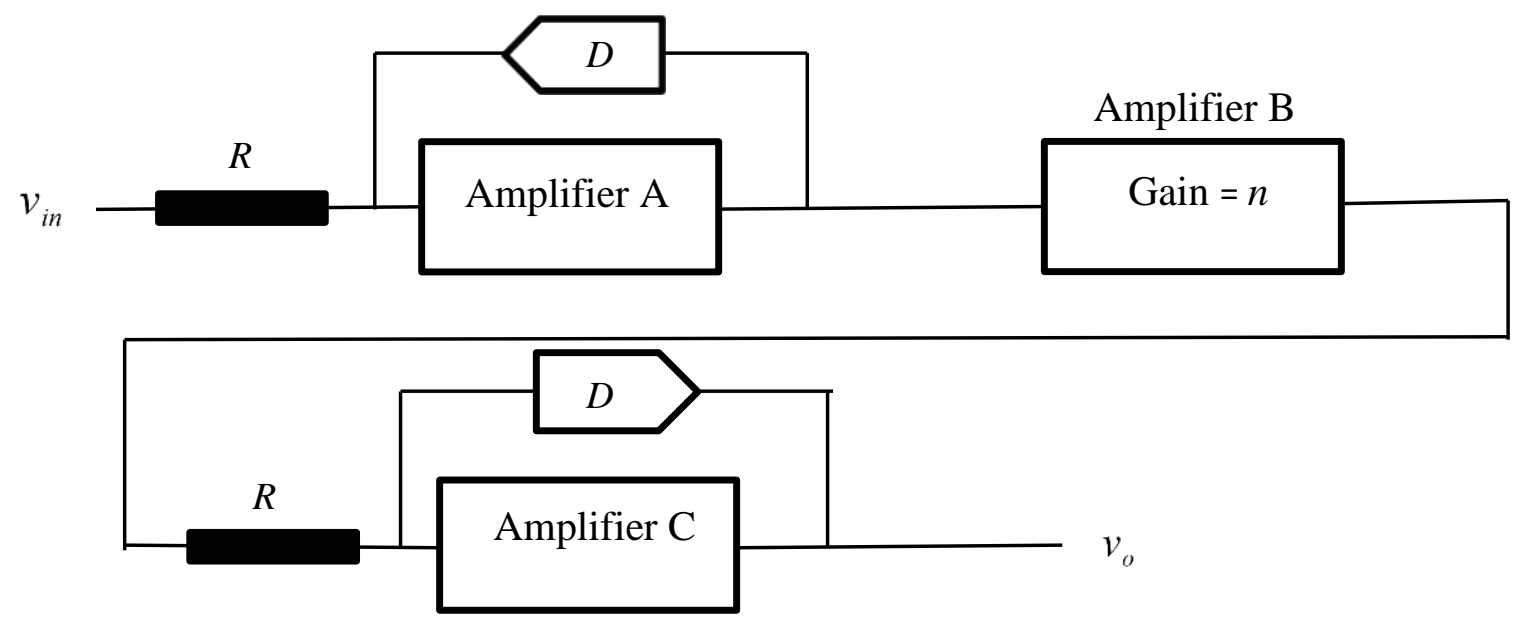

Figure (2): A circuit diagram for a unit whose input-output relationship is $v_{0}=v_{i}^{n}$

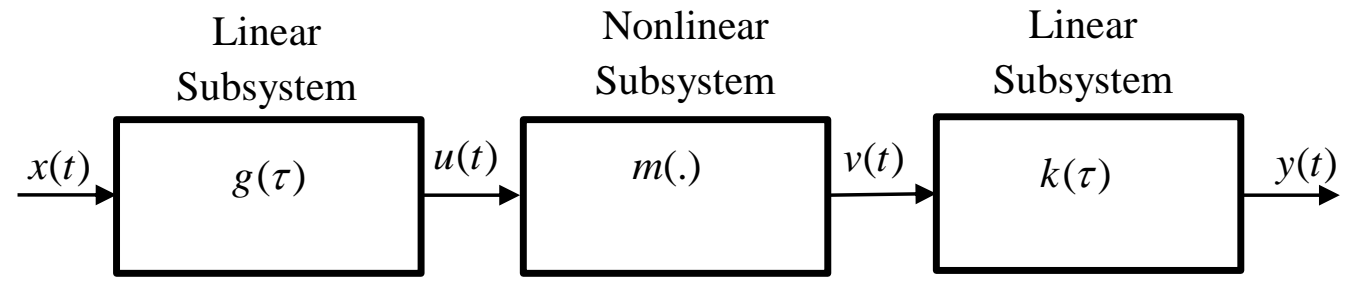

Figure (3): A cascaded subsystems of linear and nonlinear systems to compose a $\mathcal{L}-\aleph-\mathcal{L}$ system.

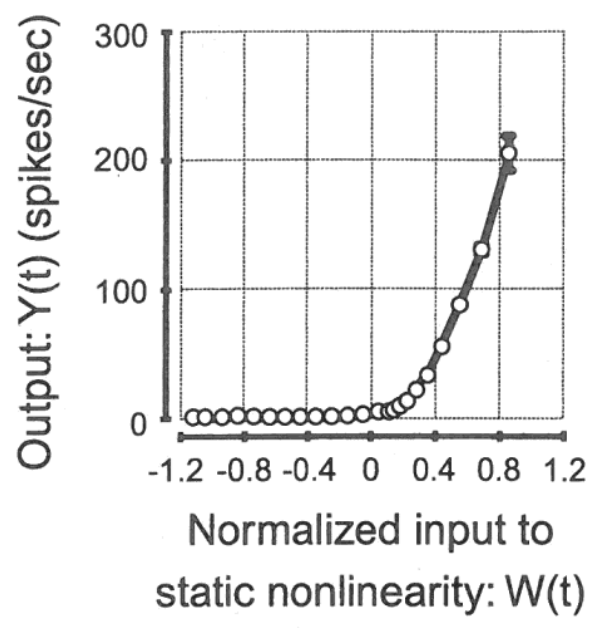

Figure (4): Biologically recorded input-output relationship according to [DeAngelis Anzai 2004] 


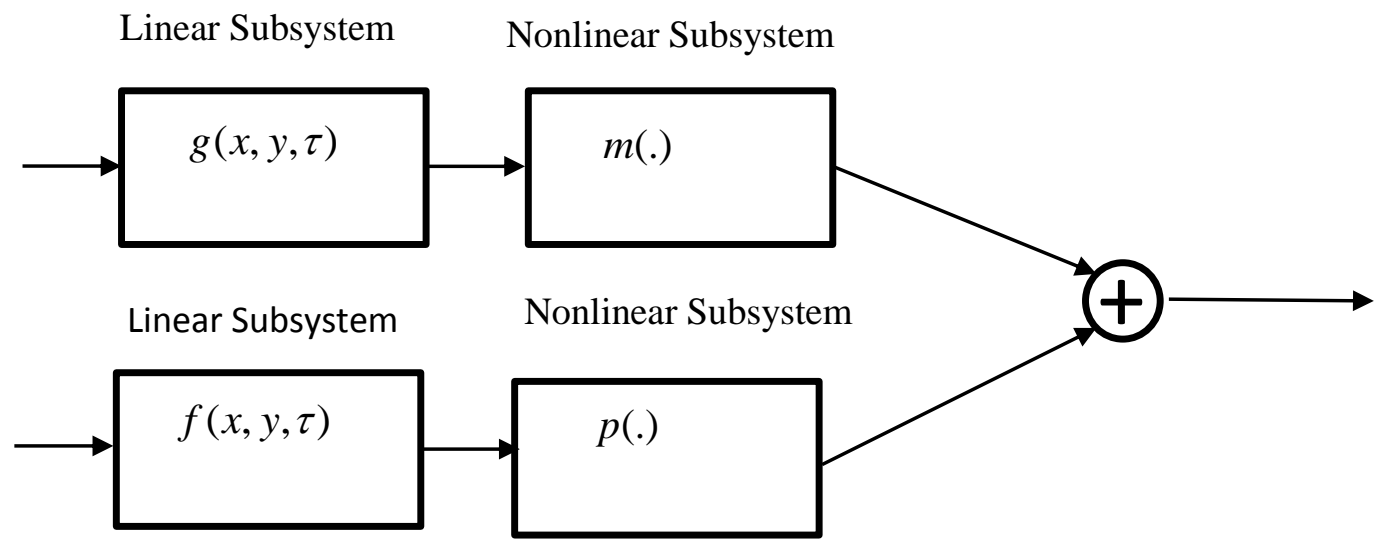

Figure (5): The structure of a complex cell containing parallel linear and nonlinear subsystems.

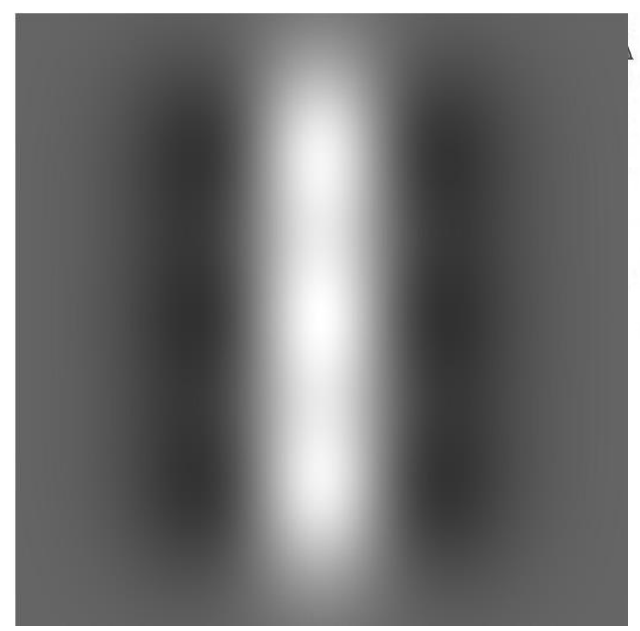

$a$

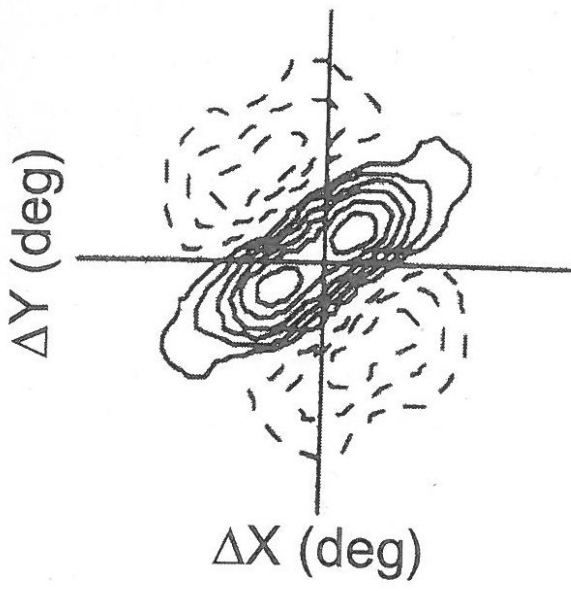

$b$

Figure (6): a) The second order responses of a complex cell whose inputs are from the simple neurons arranged as the red circles in figure (1-top ) calculated by using equation (21), b) The second order response of a V1 complex cell from a monkey reported in biological literature (DeAngelis and Anzai 2004) 


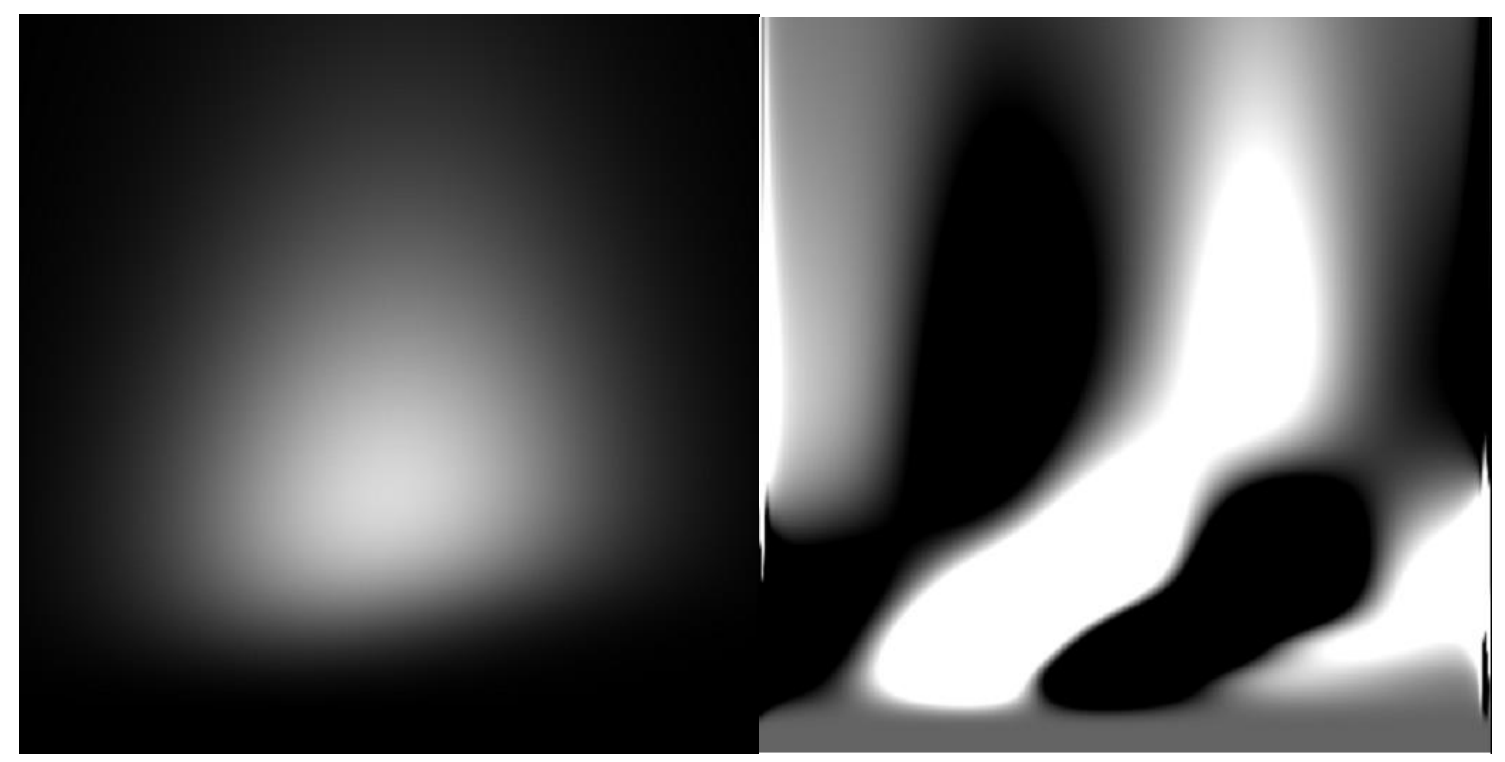

$a$

$b$
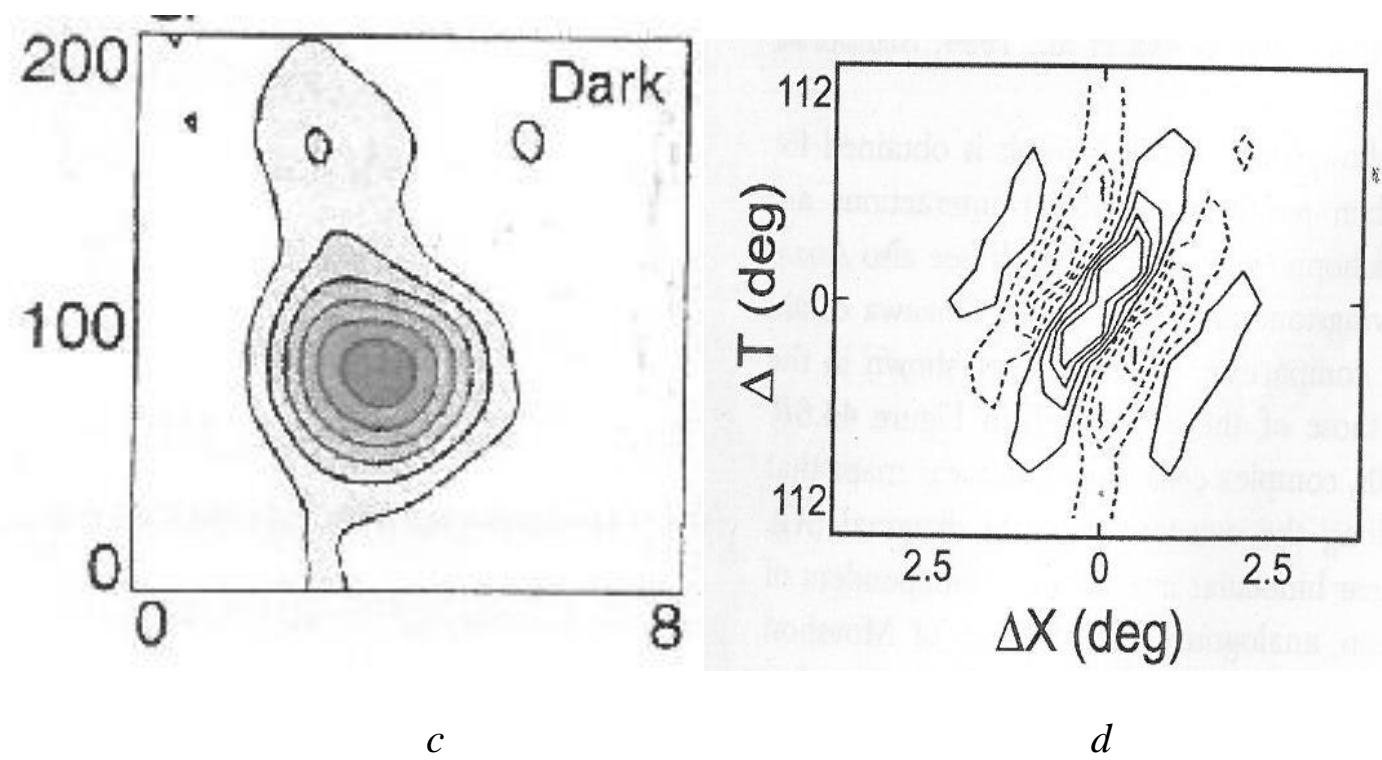

$d$

Figure (7): (a) The first order spatio-temporal response of the complex cell model $(b)$ the second order spatio-temporal response of the complex cell model based on equations (29)(32) for $n=10, R_{z} C_{z} z^{2}=800$ and $\frac{G_{z}}{C_{z}}=0.01,(c$ and $d)$ the first and second order spatiotemporal responses of a complex cell recorded in biological experiments (DeAngelis and Anzai 2004). 


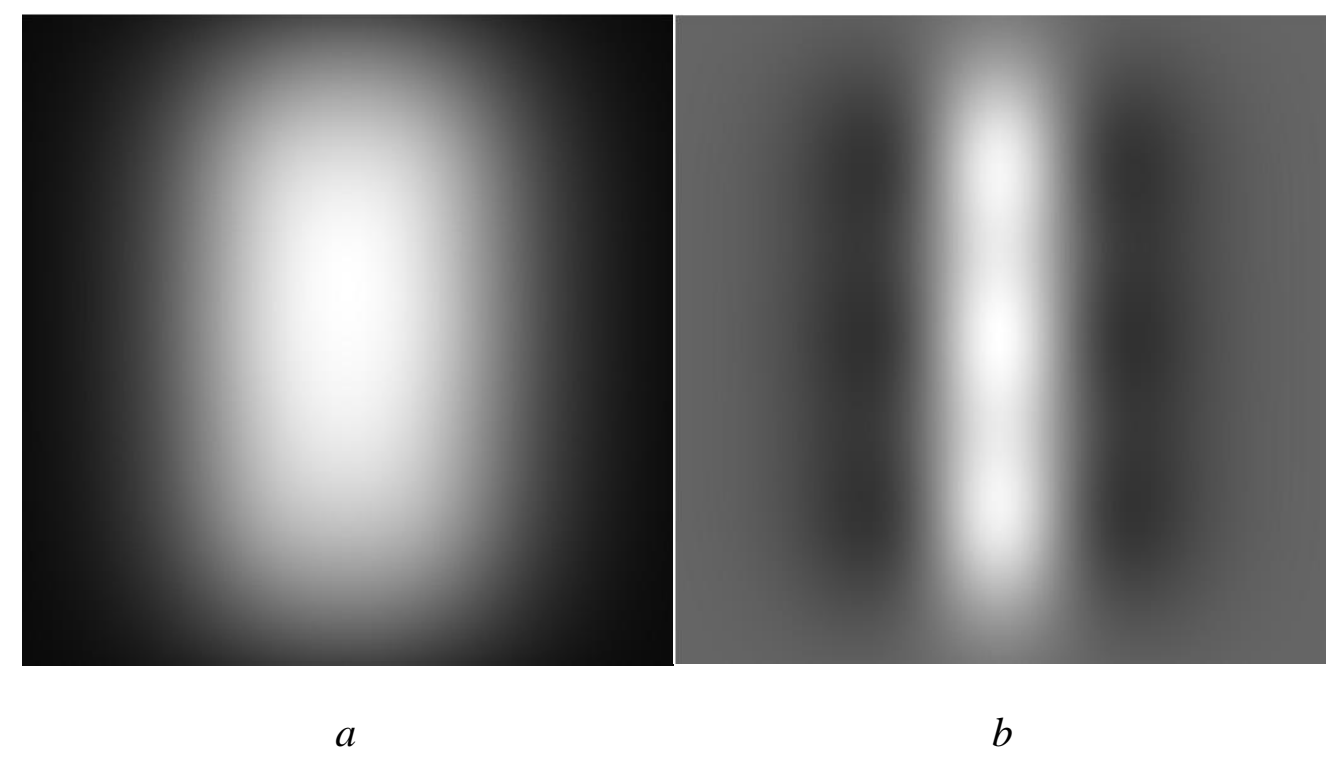

Figure(8): $a$ ) the first order and $b$ ) the second order spatial responses of a complex cell in the model proposed here. The simple cells whose outputs are input to the complex cell, are arranged as the red circles in figure (1-top). 

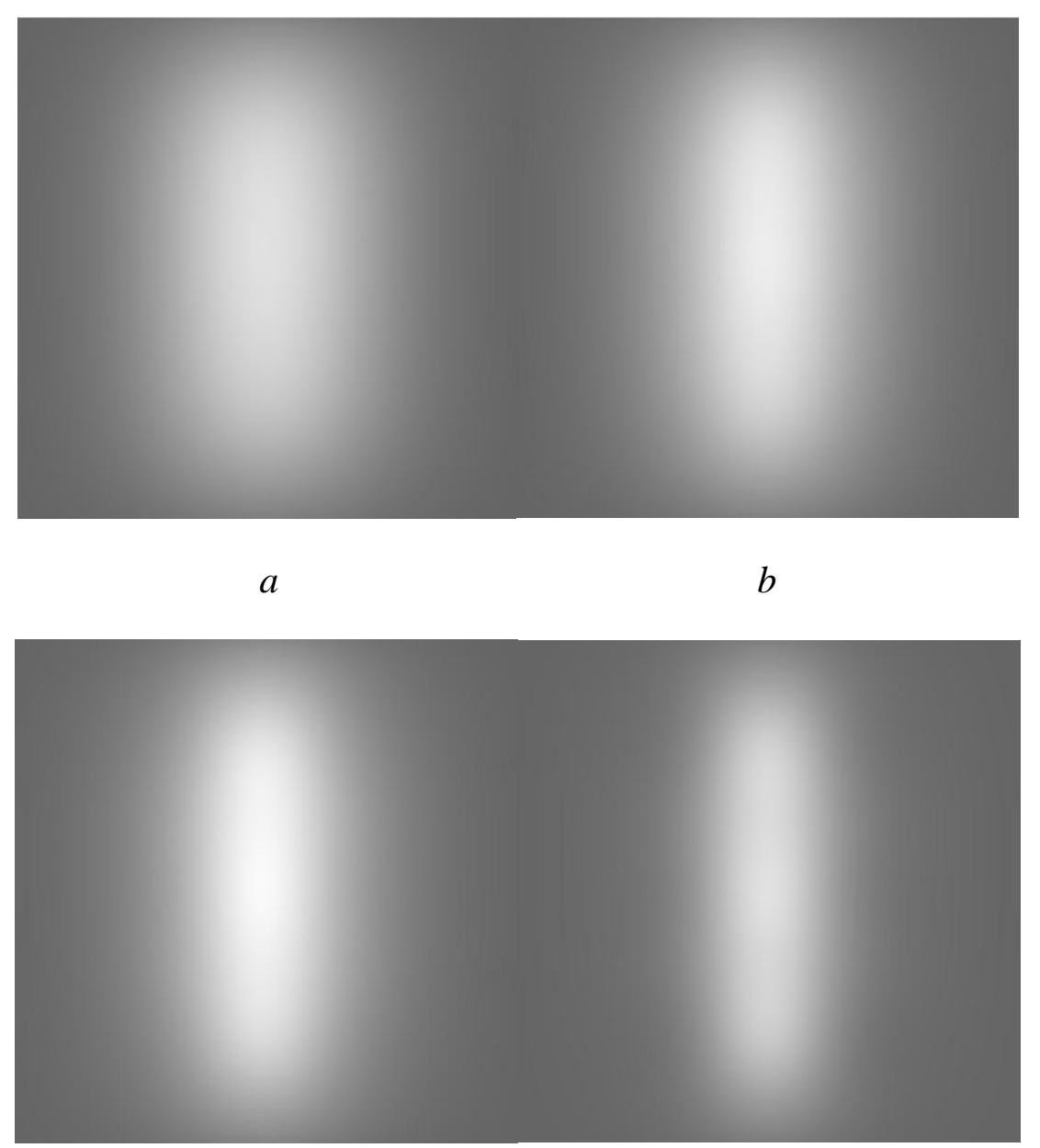

$c$

$d$

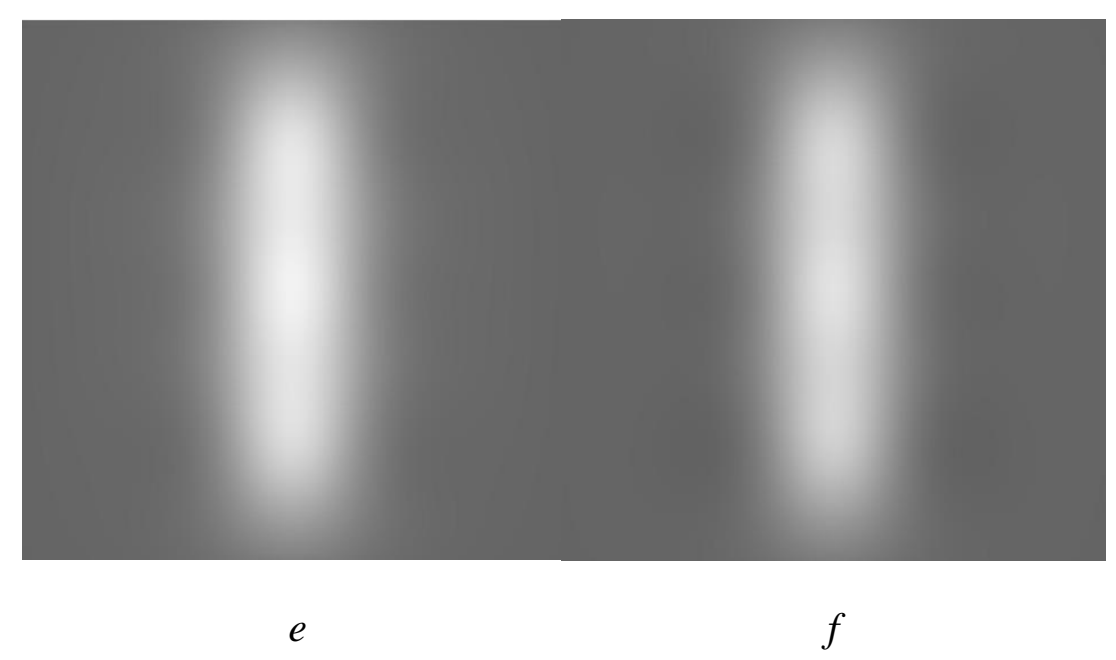

Figure (9): The first order spatial response of the complex cell model based on equations (29)-(32) for $n=10$ (after ten layers), for a) $B_{m 1}>>B_{p 1}$ b) $B_{m 1}=10 B_{p 1}$, c) $B_{m 1}=5 B_{p 1}$, d) $B_{m 1}=2 B_{p 1}$, e) $B_{m 1}=\frac{10}{7} B_{p 1}$ and f) $B_{m 1}=B_{p 1}$ 

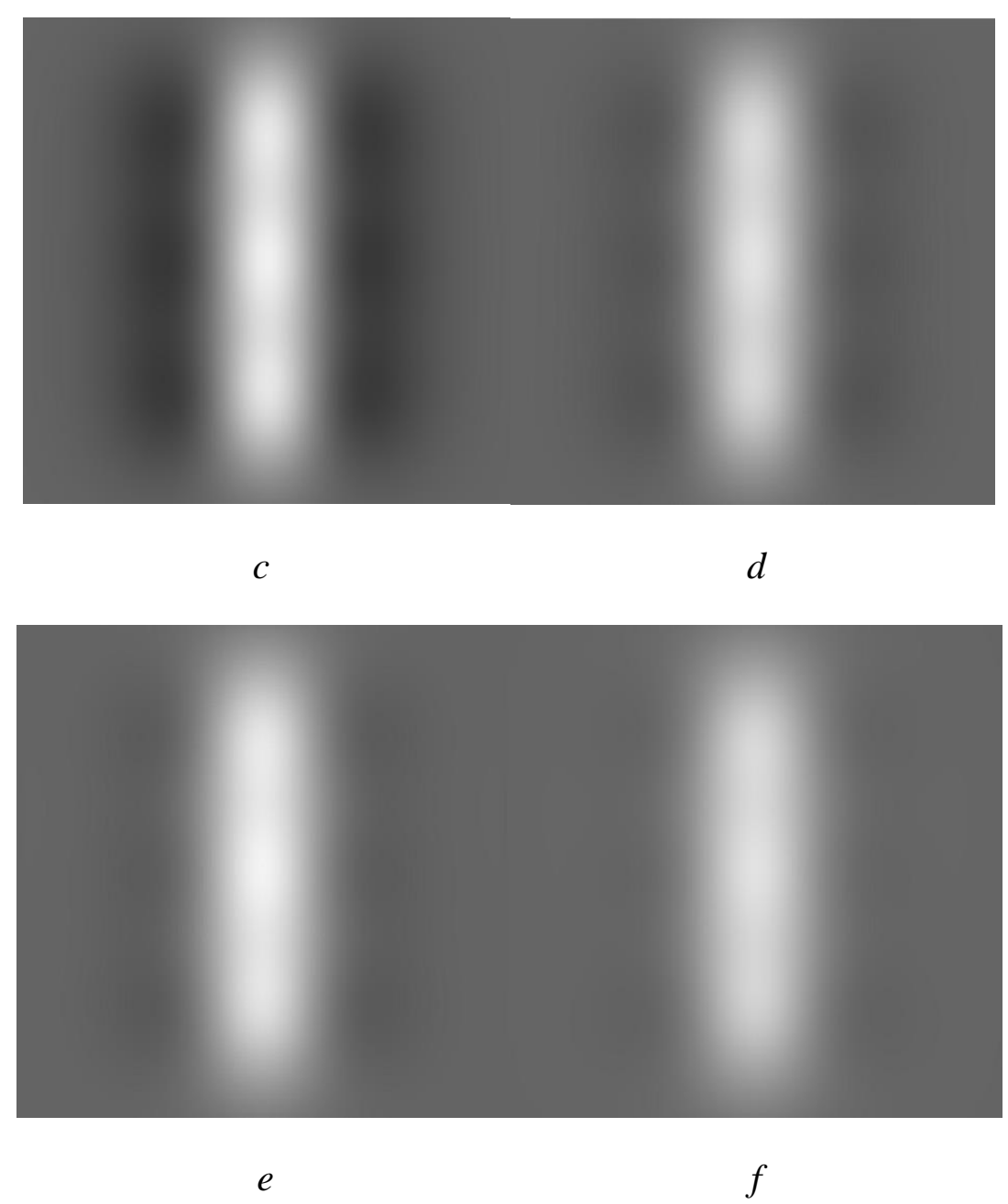

Figure (10): The second order spatial response of the complex cell model based on equations (29)-(32) for $n=10$, for a) $B_{m 2}<<B_{p 2}$ b) $B_{m 2}=\frac{B_{p 2}}{10}$, c) $B_{m 2}=\frac{B_{p 2}}{5}$, d) $B_{m 2}=\frac{B_{p 2}}{2}$, e) $B_{m 2}=\frac{7 B_{p 2}}{10}$ and f) $B_{m 2}=B_{p 2}$ 
$a$

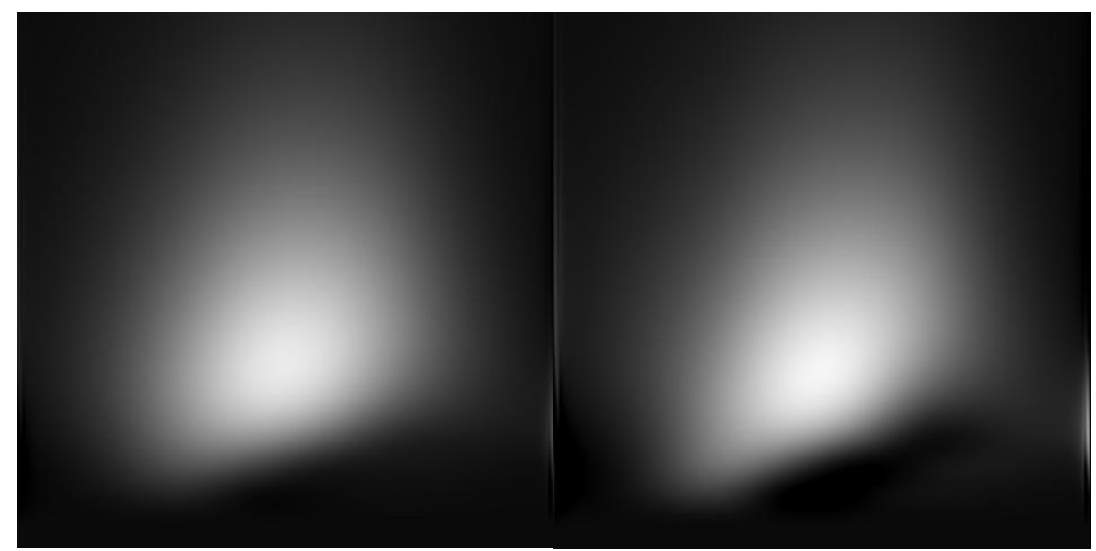

$c$

$d$

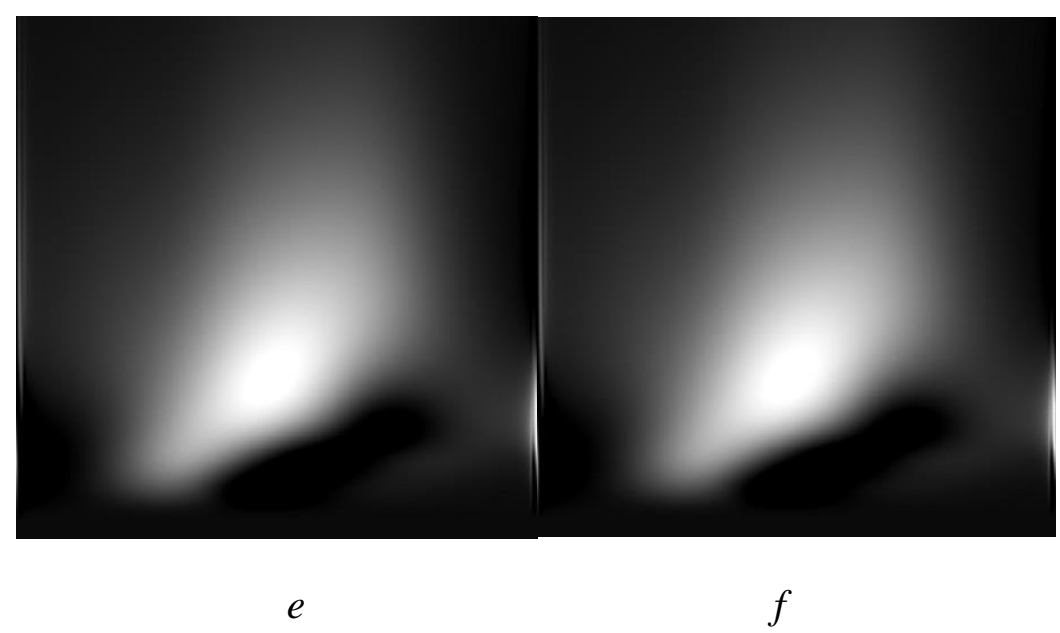

Figure (11): The first order spatio-temporal response of the complex cell model based on equations (29)-(32) for $n=10, \quad R_{z} C_{z} z^{2}=800$ and $\frac{G_{z}}{C_{z}}=0.01$, for a) $B_{m 1}>>B_{p 1}$ b) $B_{m 1}=10 B_{p 1}$, c) $B_{m 1}=5 B_{p 1}$, d) $B_{m 1}=2 B_{p 1}$, e) $B_{m 1}=\frac{10}{7} B_{p 1}$ and f) $B_{m 1}=B_{p 1}$ 


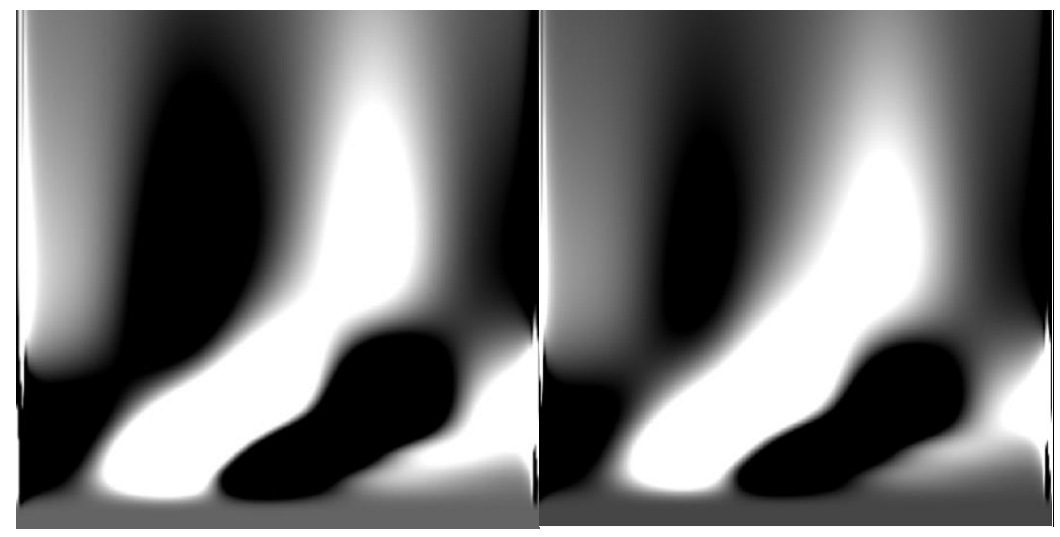

a

b

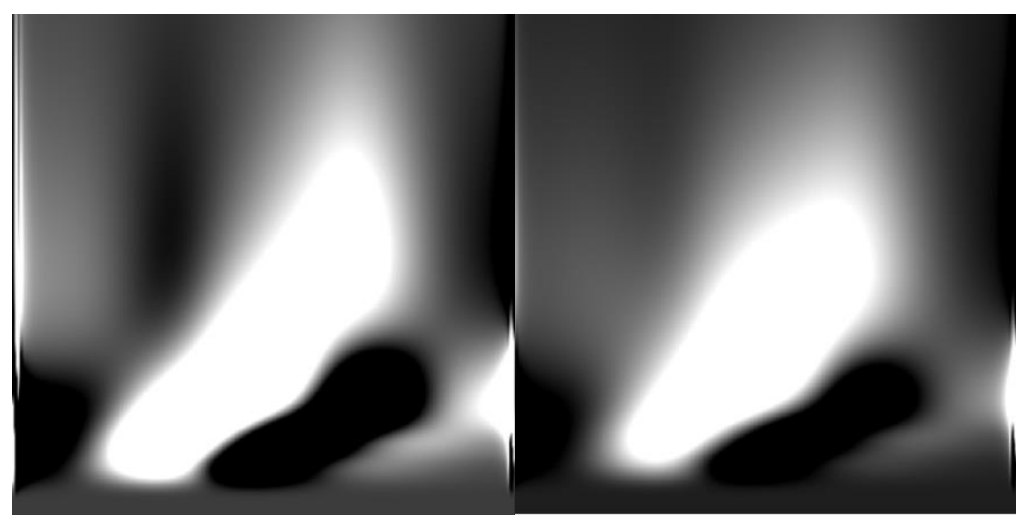

c

$d$

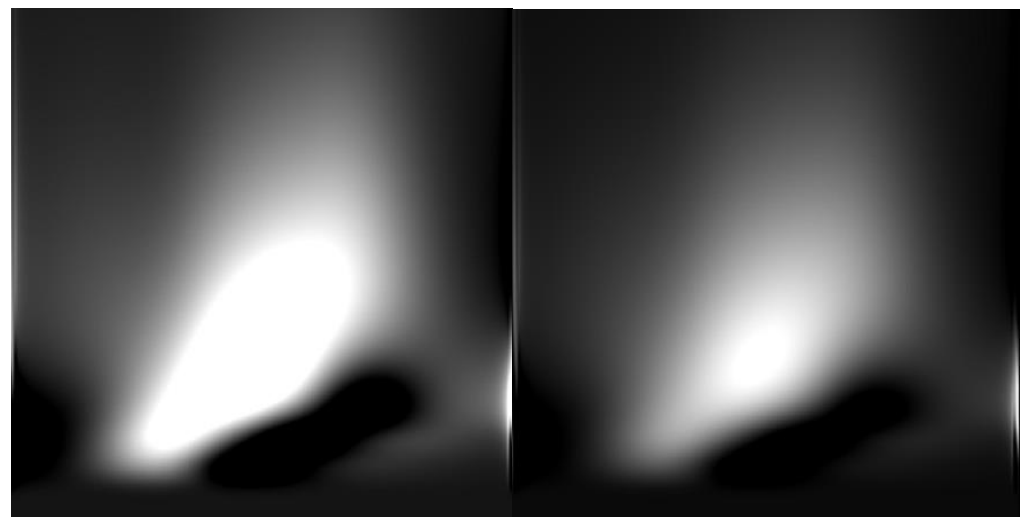

e

$f$

Figure (12): The second order spatio-temporal response of the complex cell model based on equations (29)-(32) for $n=10, \quad R_{z} C_{z} z^{2}=800$ and $\frac{G_{z}}{C_{z}}=0.01$, for a) $B_{m 2}<<B_{p 2}$ b) $B_{m 2}=\frac{B_{p 2}}{10}$, c) $B_{m 2}=\frac{B_{p 2}}{5}$, d) $B_{m 2}=\frac{B_{p 2}}{2}$, e) $B_{m 2}=\frac{7 B_{p 2}}{10}$ and f) $B_{m 2}=B_{p 2}$ 